\title{
Double Dissociation: Circadian Off-Peak Times Increase Emotional Reactivity; Aging Impairs Emotion Regulation Via Reappraisal
}

\author{
Adrienne M. Tucker, \\ Cognitive Neuroscience Division, Taub Institute for Research in Alzheimer's Disease and the \\ Aging Brain, College of Physicians and Surgeons, Columbia University
}

Rebecca Feuerstein, Cognitive Neuroscience Division, Taub Institute for Research in Alzheimer's Disease and the Aging Brain, College of Physicians and Surgeons, Columbia University

\section{Peter Mende-Siedlecki,} Department of Psychology, Princeton University

Kevin N. Ochsner, and Department of Psychology, Columbia University

Yaakov Stern Cognitive Neuroscience Division, Taub Institute for Research in Alzheimer's Disease and the Aging Brain, College of Physicians and Surgeons, Columbia University

\section{Abstract}

This study explored how the effectiveness of specific emotion regulation strategies might be influenced by aging and by time of day, given that in older age the circadian peak in cognitive performance is earlier in the day. We compared the benefit gained by 40 older (60-78 years; 20 women) and 40 younger (18-30 years; 20 women) adults during either on-peak or off-peak circadian times on 2 specific types of cognitive emotion regulation strategies: distraction and reappraisal. Participants rated their negative emotional responses to negative and neutral images under 3 conditions: a baseline nonregulation condition, a distraction condition involving a working memory task, and a reappraisal condition that involved reinterpreting the situation displayed using specific preselected strategies. First, as hypothesized, there was a crossover interaction such that participants in each age group reported more negative reactivity at their off-peak time of day. Second, a double dissociation was observed as circadian rhythms affected only negative reactivity -with reactivity highest at off-peak times-and aging diminished reappraisal but not distraction ability or reactivity. These findings add to growing evidence that understanding the effects of aging on emotion and emotion regulation depends on taking both time of day and type of regulatory strategy into account.

\section{Keywords}

emotional reactivity; emotion regulation; cognitive reappraisal; aging; circadian rhythms

\footnotetext{
(C) 2012 American Psychological Association

Correspondence concerning this article should be addressed to Yaakov Stern, Columbia University, 630 West 168th Street, P \& S Box 16, New York, NY 10032. ys11@ columbia.edu.

None of the authors have any conflicts of interest.
} 
Although many cognitive functions decline at least modestly with age (Dennis \& Cabeza, 2008; Verhaeghen, 2011), the quality of our emotional lives improves, with the ratio of positive to negative experiences increasing over time (Carstensen et al., 2011; Charles \& Carstensen, 2007). Why might this be the case? Various reasons for the "rosy glow" of older age have been proposed, and some researchers have suggested that spared or even improved emotion regulation abilities may play a key role (e.g., Phillips, Henry, Hosie, \& Milne, 2008).

Relatively little research to date has directly examined emotion regulation in aging, but extant work has focused on two types of strategies (Urry \& Gross, 2010). The first is called attentional deployment, which involves changing one's emotional response to stimuli by either selectively attending to them or aspects of them that promote desired emotional responses or by distracting oneself by thinking about stimulus-irrelevant information. Recent research suggests that older adults effectively use selective attention to regulate emotion (e.g., focusing on positive stimuli when in a negative mood; Isaacowitz, Toner, Goren, \& Wilson, 2008), but whether older adults can effectively use distraction is unclear. The second type of strategy involves changing one's emotional response by cognitively changing its meaning. The most common example is reappraisal, which involves cognitively reinterpreting the meaning of a stimulus to alter the emotional response to it. Here, results have been somewhat equivocal. On one hand, correlational studies have shown that selfreported reappraisal frequency predicts greater positive emotion (Yeung, Wong, \& Lok, 2011) or lesser autonomic arousal to aversive stimuli (Li, Fung, \& Isaacowitz, 2011). On the other hand, experimental studies have shown that in reappraisal tasks that have the goal of decreasing negative emotion, older adults are less able to reduce negative affect and show lesser activation of cognitive control-related prefrontal regions (Opitz, Rauch, Terry, \& Urry, in press; Shiota \& Levenson, 2009; Winecoff, LaBar, Madden, Cabeza, \& Huettel, 2011).

In the present study, we sought to extend prior work by examining the effects of aging on the ability to use two strategies, reappraisal and distraction, to diminish negative emotion. Aging might differentially impact these two strategies given that reappraisal and distraction seem to depend on distinct, albeit overlapping, processes (McRae et al., 2010), and that prior experimental work suggests that older adults may be impaired at reappraisal but effective at using selective attention (which is related to distraction) when attempting to decrease negative emotion (Isaacowitz et al., 2008; Urry \& Gross, 2010).

To test this hypothesis, we adapted a method we have used previously to differentiate the behavioral and neural correlates of reappraisal and distraction (McRae et al., 2010). This method presents participants with aversive images in three conditions- - a baseline condition in which they respond naturally, a reappraisal condition, and a distraction condition in which participants keep in mind an irrelevant working memory load. Comparing self-reported emotion on these three trial types allowed us to make sure that age-related differences in regulatory ability are not confounded with any age-related difference in baseline emotional reactivity (i.e., apparent enhancements or impairments in the ability to decrease emotion could result from having weaker or stronger emotions in the first place; cf. Cacioppo, Berntson, Bechara, Tranel, \& Hawkley, 2010). This is important given that two factors that covary with age may impact emotional reactivity. The first was the fact that women live longer and older populations tend to have a greater proportion of women. To account for this, an equal number of women and men were included in the younger and older groups in order to address reports that women have greater negative reactivity to aversive images than do men (e.g., Bradley, Codispoti, Sabatinelli, \& Lang, 2001). The second concerned the facts that (a) younger and older adults have different circadian rhythms, with maximal alertness and cognitive performance earlier versus later in the day for older and younger 
adults, respectively (Roenneberg et al., 2004); and (b) circadian off-peak times of day have been associated with greater negative reactivity (van Eekelen, Kerkhof, \& Amsterdam, 2003; van Eekelen, Houtveen, \& Kerkhof, 2004). Prior work on aging and emotion regulation has not taken circadian rhythms into account. We did by counterbalancing testing either in the morning or afternoon. With these methods, we sought to test two hypotheses: first, that reappraisal, not distraction, might be impaired in older adults; second, that there would be a crossover interaction such that each age group would report more negative emotional reactivity at its off-peak time of day.

\section{Method \\ Participants}

Forty younger (ages 18-30 years) and 40 older participants (ages 60-78 years) completed the paradigm. ${ }^{1}$ There were an equal number of women and men in each age group. Interested participants were screened for depression using a score $<16$ on the Center for Epidemiological Studies Depression scale; older participants were additionally screened for dementia, using a Dementia Rating Scale score $\geq 135$ (Mattis, 1988). For both age groups, current and former medical and legal students and professionals were excluded; in the second data collection wave, individuals with combat exposure were also excluded.

\section{Questionnaires}

Before completing the emotion regulation task, participants completed the Emotion Regulation Questionnaire, which asks participants to rate the degree to which they regulate their emotions using two specific strategies: reappraisal (e.g., "I control my emotions by changing the way I think about the situation I'm in") and suppression (e.g., "I control my emotions by not expressing them" Gross \& John, 2003).

\section{Tasks}

We used the paradigm of an earlier functional MRI study of young women (McRae et al., 2010). Participants viewed pictures normatively rated as negative and neutral from the International Affective Picture System (Lang, Bradley, \& Cuthbert, 2001). To compare distraction and reappraisal to unregulated responding, we displayed negative pictures for 10 $\mathrm{s}$ with three preceding 3-s displays: the word decrease (the reappraisal condition), a six-letter string of letters (the distraction condition), and the word look (the nonregulation condition). When they saw the word look, participants were instructed to pay attention and respond naturally to the subsequent stimulus, allowing themselves to have whatever reaction the picture would normally evoke in them. When they saw a six-letter string (the distraction instruction), they were instructed to try to keep all six letters in mind during the picture presentation and were told that their memory for the letters would be probed directly after the presentation of each picture. For some working memory trials, we used a fixation cross instead of an image. When they saw the word decrease, participants were asked to reinterpret the situation depicted in the picture in a way that made them feel less negative about it. ${ }^{2}$ They could do this using one of three means shown to be effective in prior work with younger adults (e.g., McRae et al., 2010): interpreting the image as not real, thinking

\footnotetext{
${ }^{1}$ In the first wave of data collection, 32 participants completed the paradigm (16 old, 16 young, even numbers of women and men). Approximately 1 year later, another 48 participants completed the paradigm ( $24 \mathrm{old}, 24$ young, even numbers of women and men). The results were not significantly different between these two groups. Thus, the data are pooled here with data collective wave (first or second) retained as an independent factor in analyses.

${ }^{2}$ For the first 32 participants, the cues were attend and reinterpret. These cues were simplified to look and decrease in the second study to make it easier for the older subjects to learn the task during the practice session. The difference in cues did not change the mean ratings of negative emotion.
} 
about how the event depicted in the image might get better, and seeing the image from a new perspective.

After viewing each image, participants were asked to rate their negative emotion using a Likert scale with anchors of $1=$ not at all and $7=$ extremely. ${ }^{3}$ For the working memory trials, they next indicated whether or not they recognized a single probe letter. Three versions of the task were used to counterbalance the negative images across trial types. Each task version contained 12 trials of each combination of condition and picture valence, for a total of 72 trials.

\section{Time of Day Manipulation}

Half of all participants were run at $10 \mathrm{a} . \mathrm{m}$. and the other half were run at 2 p.m.

\section{Main Task Analyses}

Mixed-effects analyses of variance (ANOVAs) were used. In the main task model, there were six independent variables: trial type (look negative vs. look neutral), age group (young vs. old), sex (female vs. male), time of day (10 a.m. vs. 2. p.m.), task version (three equivalent task versions counterbalancing the negative stimuli across conditions), and data collection wave (first or second). There were no significant effects of either task version or data collection wave in any of the analyses; thus, these independent variables are not discussed further.

Three dependent variables were of interest. Negative emotional reactivity was computed as the difference between self-reports of emotion on look trials with negative images and look trials with neutral images. Distraction ability was computed as the difference of self-reported negative affect for look trials with negative images and working memory trials with negative images. Reappraisal ability was computed as the difference between self-reported negative affect on look trials with negative images and decrease trials (all decrease trials involved a negative image). In each model, the main effect of trial type represented the variable of interest (i.e., negative reactivity or reappraisal ability). Three separate mixed-effects ANOVAs were used to investigate each of the three response outcomes. Then, a fourth mixed-effects ANOVA was used to investigate how reappraisal ability and distraction ability compared with one another in younger and older adults. The effect of aging was captured by a two-way interaction of Age Group $\times$ Trial Type, and the effect of circadian rhythm was captured by a three-way interaction of Age Group $\times$ Trial Type $\times$ Time of Day.

\section{Results}

\section{Questionnaires}

Younger and older adults were equivalent in terms of reported levels of depressive symptoms on the Center for Epidemiological Studies Depression scale and self-reported tendencies to regulate emotion using reappraisal on the Emotion Regulation Questionnaire. On average, the younger adults had completed 1 year less of education. This was statistically significant. See Table 1.

\section{Tasks}

Negative reactivity-Significantly more negative emotion was reported for look-negative trials than for look-neutral trials, $P(1,68)=526.48, p<.0001$, indicating that our

\footnotetext{
${ }^{3}$ For the first 32 participants, the anchors were $1=$ weak and $7=$ strong. In the second study, these were changed to be clearer for the older participants. The difference in the anchor labels did not change the mean ratings of negative emotion.
} 
experimental manipulation of negative emotion was successful. The age groups had equivalent negative reactivity as revealed by the lack of a significant interaction of Age $\times$ Trial Type, $F(1,68)=0.13, p=.72$. Because younger and older participants had the same magnitude of emotional reactivity, the two age groups had the same magnitude of negative emotion available to be regulated. This is important because it means that age-related differences in the efficacy of distraction or reappraisal (see below) could not be attributed to differing strengths of emotional response in the two groups. Women reported more negative reactivity than did men, $F(1,68)=6.50, p=.01$. As hypothesized, there was a circadian effect captured by a three-way Age Group $\times$ Trial Type $\times$ Time of Day interaction such that each age group had more negative reactivity at its off-peak time of day, $F(1,68)=6.88, p=$. 01. See Figure 1.

Reappraisal ability-Overall, reappraisal significantly reduced negative emotion (i.e., main effect of trial type), $F(1,68)=77.48, p<.0001$. There was a trend for a main effect of age group, $F(1,68)=3.35, p=.07$, as well as a significant interaction of Age Group $\times$ Time of Day, $F(1,68)=4.19, p=.04$. As hypothesized, older adults received less benefit from reappraisal (i.e., interaction of Age Group $\times$ Trial Type), $F(1,68)=10.28, p=.002$. There was no circadian effect on reappraisal benefit as captured by the lack of a significant threeway interaction of Age Group $\times$ Trial Type $\times$ Time of Day, $F(1,68)=1.63, p=.21$. Thus, although overall each group had more negative emotion at its off-peak time of day pooling across both look (nonregulation) and decrease (reappraise) trials, the difference between the two-representing the ability to decrease experienced negative emotion using cognitive reappraisal—was not different as a function of time of day.

Distraction ability-Overall, there was significantly less negative emotion reported for working memory-negative trials than for look-negative trials, indicating that distraction significantly reduced negative emotion, $F(1,68)=22.26, p<.0001$. There was a trend for this effect to be bigger for women than men, $F(1,68)=3.86, p=.05$. There were no significant effects of age (i.e., no interaction between Age Group $\times$ Trial Type), $F(1,68)=$ $0.19, p=.66$ (see Figure 1) or circadian rhythm on distraction ability (i.e., no three-way interaction between Age Group $\times$ Trial Type $\times$ Time of Day), $F(1,68)=1.28, p=.26$. Working memory accuracy was lower for older adults, so analyses were repeated with correct trials only. The overall pattern of results did not differ between the two analyses.

Reappraisal ability versus distraction ability-Reappraisal reduced negative emotion significantly more than did distraction, $F(1,68)=31.50, p<.0001$. This effect was present in the younger adults, who got a significantly bigger benefit from reappraisal than from distraction, $F(1,36)=41.02, p<.0001$. In older adults, there was only a trend for a difference in benefit between reappraisal and distraction, $F(1,32)=2.94, p=.096$.

\section{Discussion}

Overall Summary

We report here the results of a study directly comparing younger and older adults at both circadian on-peak and off-peak times of day on measures of emotional reactivity and their ability to implement two specific cognitive emotion regulation strategies: distraction and reappraisal. With respect to self-reported emotional reactivity to aversive images, although there were no age-related differences, reactivity was greater for women and at the circadian off-peak time of day for each age group. Second, although both strategies were effective for both age groups, reappraisal was more effective than distraction overall, and there were agerelated differences in the efficacy with which distraction and reappraisal were deployed: Although distraction was equally effective for both groups, older adults were less able than 
younger adults to reduce their negative affect via reappraisal. Taken together, these findings show a double dissociation between the variables that impact emotional reactivity as opposed to emotion regulation.

\section{Age Differences in Emotion Regulation}

In general, the present findings are consistent with an emerging view that age impacts emotion regulation ability more than it impacts emotional reactivity (Nashiro, Sakaki, \& Mather, 2012). More specifically, the present findings are consistent with prior work suggesting that the ability to regulate emotion using attentional strategies-such as selective attention (Isaacowitz et al., 2008) or distraction, as studied here-may be intact or even enhanced in aging, whereas the ability to cognitively change the meaning of a stimulus via reappraisal may be impaired. Whether reappraisal is impaired in all cases remains to be seen, however. On one hand, the present results fit with three prior studies showing that older adults are less able to use reappraisal to diminish negative emotion (Opitz et al., in press; Shiota \& Levenson, 2009; Winecoff et al., 2011). On the other hand, there are reports that older adults may be better able to use reappraisal to increase negative emotion (Opitz et al., in press) or use variants of reappraisal that focus on up-regulating positive emotion (Shiota \& Levenson, 2009). Future work may determine whether apparent age-related impairments depend on the specific goals that guide reappraisal and tactics used to reappraise. Clarifying these issues is important, given that reappraisal is the only strategy tied to lower vulnerability for long-term psychiatric symptoms in younger and older adults (e.g., Kross \& Ayduk, 2008). Thus, reappraisal may be one key target of intervention for building resilience in later life (cf. Troy \& Mauss, 2011).

\section{Circadian and Gender Differences in Emotional Reactivity}

Although self-reported reactivity did not differ as a function of age, we did find that it was greater at circadian off-peak times and in women. In neither case did greater reactivity interact with regulatory ability, suggesting that the effects of circadian rhythm and gender on emotion generation are independent of the effects of aging on regulatory ability.

The finding that younger adults reported more negative reactivity in the morning whereas older adults reported more negative reactivity in the afternoon fits with prior work showing similar circadian off-peak effects (van Eekelen et al., 2003, 2004). Although the magnitude of the reported emotion decrease using the two emotion regulation strategies was not affected by circadian factors, given that the measurement of reported negative emotion was influenced by the time of day of assessment and that this differed as a function of age, it may nonetheless be important that future work take circadian factors into account when comparing emotional responding in younger and older adults. Circadian confounds are most likely for studies without explicit instructions as to whether or how to regulate as well as for studies that are interested in estimating the magnitude of negative reactivity. Such confounds are less likely for studies in which the focus is on emotion regulation and explicit instructions are provided as to when and how to regulate.

Negative reactivity was also greater in women, as has been reported previously (e.g., Bradley et al., 2001). However, this effect is not always found, and in some cases gender differences in regulatory ability are found instead (e.g., McRae, Ochsner, Mauss, Gabrieli, \& Gross, 2008). Although it is not immediately apparent why such gender differences are or are not found in specific studies, it remains for future work to determine whether variables such as the specific regulatory strategy or stimuli used determine whether gender differences will be observed. 


\section{Limitations}

A chief limitation of this study is that emotion was probed solely through self-report. The reliance on self-report measures leaves open the possibility that demand characteristics contributed to the findings reported here. We attempted to minimize demand characteristics during instruction by emphasizing that participants might not feel negative emotion in response to all images and further that the strategies provided might not always be effective in reducing negative emotion.

A second limitation is that circadian rhythms were probed through groups of older versus younger adults who on average are morning-type versus evening-type individuals, respectively. Another strategy that has often been used is to prescreen to select older adults who are morning type and only include younger adults who are evening type (e.g., Yoon, May, \& Hasher, 1999). Although powerful, this method has the limitation of excluding more younger than older adults. That said, given that we and others (West, Murphy, Armilio, Craik, \& Stuss, 2002) observed a crossover interaction of age by time of day even without preselecting subjects on the basis of chronotype, the effects of aging on advancing the circadian rhythm seem to be large enough that prescreening is not necessary (cf. May, Hasher, \& Stoltzfus, 1993).

\section{Final Summary}

In summary, a double dissociation was obtained as circadian off-peak times of day amplified only negative emotional reactivity, whereas aging impaired only cognitive reappraisal. Distraction was unaffected by circadian rhythm or aging. These findings add to growing evidence that understanding the effects of aging on emotion and emotion regulation depends on taking both time of day and type of regulatory strategy into account.

\section{Acknowledgments}

This work was supported by National Institute of Aging Grants T32 AG00261 and R01 AG026158 to Yaakov Stern and by National Institutes of Health Grant MH076137 to Kevin N. Ochsner.

\section{References}

Bradley MM, Codispoti M, Sabatinelli D, Lang PJ. Emotion and motivation: II. Sex differences in picture processing. Emotion. 2001; 1:300-319. doi:10.1037/1528-3542.1.3.300. [PubMed: 12934688]

Cacioppo, JT.; Berntson, GG.; Bechara, A.; Tranel, D.; Hawkley, LC. Could an aging brain contribute to subjective well-being? The value added by a social neuroscience perspective. In: Todorov, A.; Fiske, S.; Prentice, D., editors. Social neuroscience: Toward understanding the underpinnings of the social mind. Oxford University Press; New York, NY: 2010. p. 249-262.

Carstensen LL, Turan B, Scheibe S, Ram N, Ersner-Hershfield H, Samanez-Larkin GR. Emotional experience improves with age: Evidence based on over 10 years of experience sampling. Psychology and Aging. 2011; 26:21-33. doi:10.1037/a0021285. [PubMed: 20973600]

Charles, ST.; Carstensen, LL. Emotion regulation and aging. In: Gross, JJ., editor. The handbook of emotion regulation. Guilford Press; New York, NY: 2007. p. 307-327.

Dennis, NA.; Cabeza, R. Neuroimaging of healthy cognitive aging. In: Craik, FIM.; Salthouse, TA., editors. The handbook of aging and cognition. Psychology Press; New York, NY: 2008. p. 1-54.

Gross JJ, John OP. Individual differences in two emotion regulation processes: Implications for affect, relationships, and wellbeing. Journal of Personality and Social Psychology. 2003; 85:348-362. doi: 10.1037/0022-3514.85.2.348. [PubMed: 12916575]

Isaacowitz DM, Toner K, Goren D, Wilson HR. Looking while unhappy: Mood-congruent gaze in young adults, positive gaze in older adults. Psychological Science. 2008; 19:848-853. doi:10.1111/ j.1467-9280.2008.02167.x. [PubMed: 18947348] 
Kross E, Ayduk O. Facilitating adaptive emotional analysis: Distinguishing distanced-analysis of depressive experiences from immersed-analysis and distraction. Personality and Social Psychology Bulletin. 2008; 34:924-938. doi:10.1177/0146167208315938. [PubMed: 18469151]

Lang, PJ.; Bradley, MM.; Cuthbert, BN. International Affective Picture System (IAPS). Instruction manual and affective ratings (Tech. Rep.). University of Florida, Center for Research in Psychophysiology; Gainesville, FL: 2001.

Li T, Fung HH, Isaacowitz DM. The role of dispositional reappraisal in the age-related positivity effect. The Journals of Gerontology, Series B: Psychological and Social Sciences. 2011; 66:56-60. doi: $10.1093 /$ geronb/gbq074.

Mattis, S. Dementia Rating Scale (DRS). Psychological Assessment Resources; Odessa, FL: 1988.

May CP, Hasher L, Stoltzfus ER. Optimal time of day and the magnitude of age differences in memory. Psychological Science. 1993; 4:326-330. doi:10.1111/j.1467-9280.1993.tb00573.x.

McRae K, Hughes B, Chopra S, Gabrieli JDE, Gross JJ, Ochsner KN. The neural bases of distraction and reappraisal. Journal of Cognitive Neuroscience. 2010; 22:248-262. doi:10.1162/jocn. 2009.21243. [PubMed: 19400679]

McRae K, Ochsner KN, Mauss IB, Gabrieli JJD, Gross JJ. Gender differences in emotion regulation: An fMRI study of cognitive reappraisal. Group Processes \& Intergroup Relations. 2008; 11:143162. doi:10.1177/1368430207088035.

Nashiro K, Sakaki M, Mather M. Age differences in brain activity during emotion processing: Reflections of age-related decline or increased emotion regulation? Gerontology. 2012; 58:156163. doi:10.1159/000328465. [PubMed: 21691052]

Opitz PC, Rauch LC, Terry DP, Urry HL. Prefrontal mediation of age differences in cognitive reappraisal. Neurobiology of Aging. in press.

Phillips LH, Henry JD, Hosie JA, Milne AB. Effective regulation of the experience and expression of negative affect in old age. The Journals of Gerontology, Series A: Biological Sciences and Medical Sciences. 2008; 63:P138-P145.

Roenneberg T, Kuehnle T, Pramstaller PP, Ricken J, Havel M, Guth A. A marker for the end of adolescence. Current Biology. 2004; 14:R1038-R1039. doi:10.1016/j.cub.2004.11.039. [PubMed: 15620633]

Shiota MN, Levenson RW. Effects of aging on experimentally instructed detached reappraisal, positive reappraisal, and emotional behavior suppression. Psychology and Aging. 2009; 24:890900. doi:10.1037/a0017896. [PubMed: 20025404]

Troy, AS.; Mauss, IB. Resilience in the face of stress: Emotion regulation as a protective factor. In: Southwick, S.; Charney, D.; Friedman, M.; Litz, B., editors. Resilience and mental health: Challenges across the lifespan. Cambridge University Press; New York, NY: 2011. p. 30-44.doi: 10.1017/CBO9780511994791.004

Urry HL, Gross JJ. Emotion regulation in older age. Current Directions in Psychological Science. 2010; 19:352-357. doi:10.1177/0963721410388395.

van Eekelen APJ, Houtveen JH, Kerkhof GA. Circadian variation in cardiac autonomic activity: Reactivity measurements to different types of stressors. Chronobiology International. 2004; 21:107-129. doi:10.1081/CBI-120027983. [PubMed: 15129827]

van Eekelen APJ, Kerkhof GA, Amsterdam JGC. Circadian variation in cortisol reactivity to an acute stressor. Chronobiology International. 2003; 20:863-878. doi:10.1081/CBI-120024212. [PubMed: 14535359]

Verhaeghen P. Aging and executive control: Reports of a demise greatly exaggerated. Current Directions in Psychological Science. 2011; 20:174-180. doi:10.1177/0963721411408772.

West R, Murphy KJ, Armilio ML, Craik FIM, Stuss DT. Effects of time of day on age differences in working memory. The Journals of Gerontology, Series B: Psychological and Social Sciences. 2002; 57:3-10.

Winecoff A, LaBar KS, Madden DJ, Cabeza R, Huettel SA. Cognitive and neural contributors to emotion regulation in aging. Social Cognitive and Affective Neuroscience. 2011; 6:165-176. doi: 10.1093/scan/nsq030. [PubMed: 20385663]

Yeung DY, Wong CK, Lok DP. Emotion regulation mediates age differences in emotions. Aging \& Mental Health. 2011; 15:414-418. doi:10.1080/13607863.2010.536136. [PubMed: 21491227] 
Yoon, C.; May, CP.; Hasher, L. Aging, circadian arousal patterns, and cognition. Psychology Press; Philadelphia, PA: 1999. 

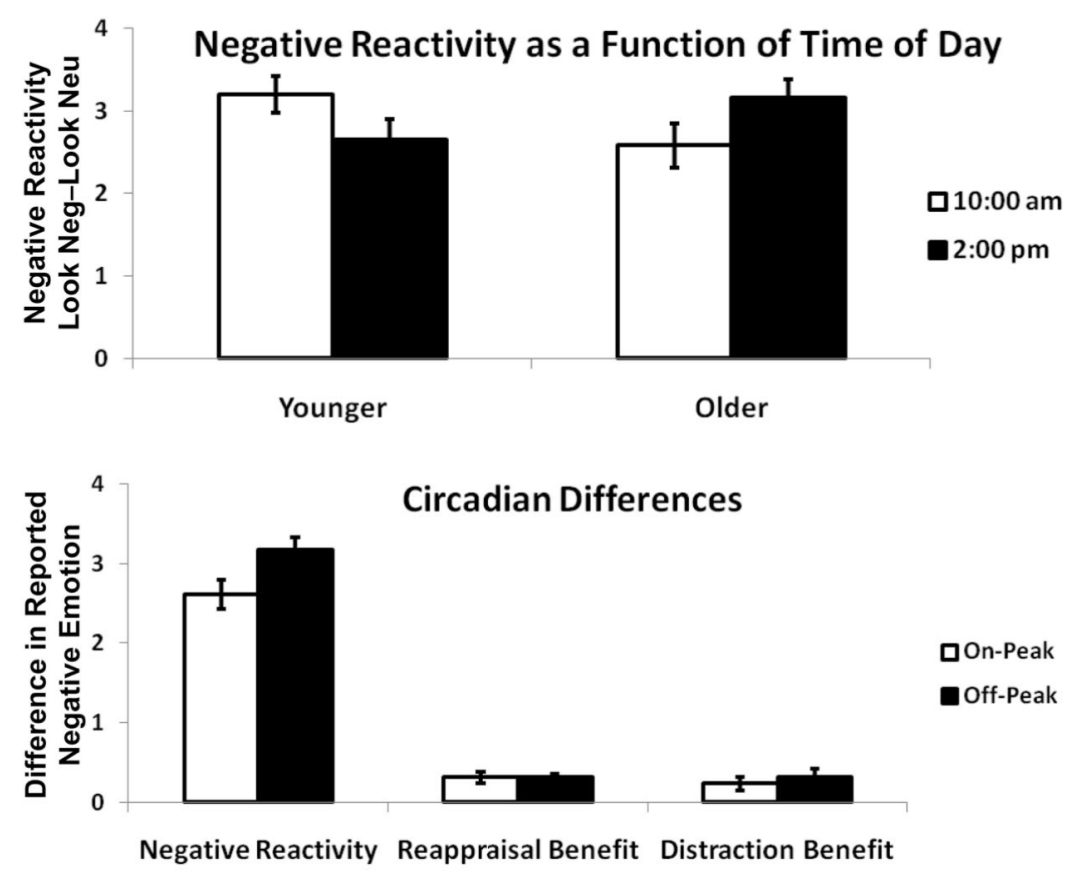

口On-Peak

口Off-Peak

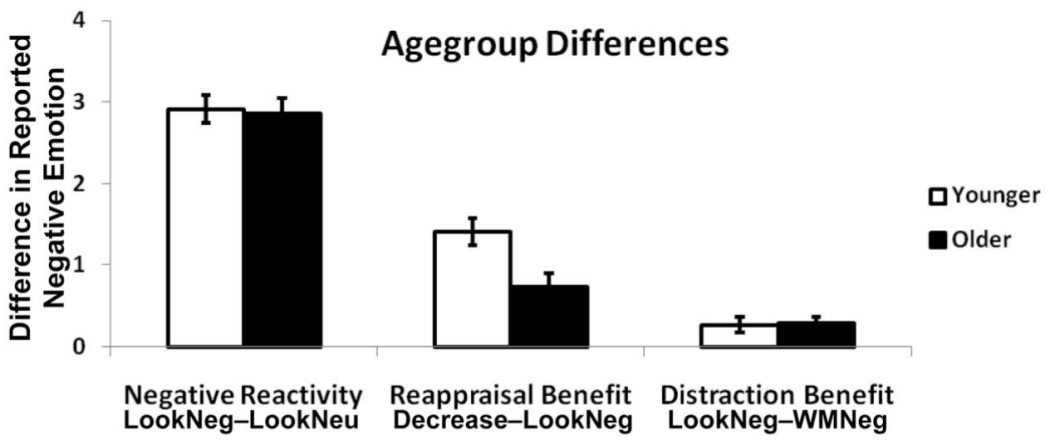

Figure 1.

Circadian and aging effects on outcome variables. The top panel represents time of day effects on negative reactivity for younger and older adults separately, displaying the crossover interaction. The middle panel represents the summary of circadian off-peak times of day affecting only negative reactivity, and the bottom panel represents the summary of aging affecting only reappraisal. The raw difference scores representing the three outcome variables of interest are displayed, excluding outliers. Columns represent the raw mean \pm standard error. 
Table 1

Demographic Variables by Age Group

\begin{tabular}{lcc}
\hline \multicolumn{1}{c}{ Variable } & Younger & Older \\
\hline $\begin{array}{l}\text { Mean }(S E) \text { age (years) }{ }^{*} \\
\text { Female, \% }\end{array}$ & $23.5(0.6)$ & $67.4(0.6)$ \\
Mean $(S E)$ depression screen score & 50.0 & 50.0 \\
$\quad(>15$ may indicate mild depression) & $6.4(0.7)$ & $6.5(0.8)$ \\
Mean $(S E)$ Emotion Regulation & & \\
$\quad \begin{array}{l}\text { Questionnaire score (reported use of } \\
\text { reappraisal) }\end{array}$ & $30.2(1.0)$ & $29.8(1.2)$ \\
Mean $(S E)$ education (years) & & \\
\hline * & $15.7(0.3)$ & $16.8(0.4)$ \\
$p<.05$. & &
\end{tabular}

